\title{
The local existence of strong solution for the stochastic 3D Boussinesq equations
}

Lihuai $\mathrm{Du}^{1,2^{*}}$ (D)

\section{"Correspondence:}

dulihuai@zju.edu.cn

${ }^{1}$ Institute of Applied Physics and

Computational Mathematics,

Beijing, P.R. China

${ }^{2}$ School of Mathematical Sciences,

Zhejiang University, Hangzhou,

P.R. China

\section{Springer}

\begin{abstract}
The stochastic 3D Boussinesq equations with additive noise are considered. We prove the local existence of the strong solution in $H^{s}\left(\frac{1}{2}<s \leq 1\right)$. We also obtain a new stopping time, which shows that the ${\mathrm{H}^{\frac{1}{2}}}^{+}$norm of $u$ controls the breakdown of the strong solution. Furthermore, we give the probability estimate of the lifespan larger than $\delta(0<\delta<1)$.
\end{abstract}

MSC: Primary 60H15; secondary 35Q35; 35R60; 76D03

Keywords: Stochastic Boussinesq equation; Strong solution; Stopping time estimate

\section{Introduction}

In this paper, we consider the stochastic 3D Boussinesq equations driven by an additive noise:

$$
\left\{\begin{array}{l}
d u+(u \cdot \nabla u-v \Delta u+\nabla p) d t=\rho e_{3} d t+\sum_{i=1}^{\infty} \Phi_{1, i} d W_{i}, \\
d \rho+(u \cdot \nabla \rho-\kappa \Delta \rho) d t=\sum_{i=1}^{\infty} \Phi_{2, i} d \widetilde{W}_{i}, \quad(t, x) \in\left(\mathbb{R}^{+} \times \mathbb{R}^{3}\right), \\
\nabla \cdot u=0, \\
\left.(u, \rho)\right|_{t=0}=\left(u_{0}, \rho_{0}\right),
\end{array}\right.
$$

where $u=\left(u_{1}, u_{2}, u_{3}\right)$ is the velocity field of the flow, $\rho$ is the scalar temperature, and $p$ is the scalar pressure; $v, \kappa$ are nonnegative viscosity parameters and $e_{3}$ is the vertical unit vector of $\mathbb{R}^{3} \cdot\left\{W_{i}\right\}_{i=1}^{\infty},\left\{\widetilde{W}_{i}\right\}_{i=1}^{\infty}$ are given independent standard Brownian motions defined in the filtered space $\left(\Omega, \mathcal{F}, \mathcal{F}_{t}, \mathbb{P}\right.$ ) with $\mathcal{F}_{t}$ (a set of sub $\sigma$-fields of $\mathcal{F}$ with $\mathcal{F}_{s} \subset \mathcal{F}_{t} \subset \mathcal{F}$ if $0 \leq s<t<\infty), \Phi_{i, 1}$ and $\Phi_{i, 2}$ are the components of the random force.

If $\Phi_{i, 1}=\Phi_{i, 2} \equiv 0$, system (1.1) becomes the deterministic Boussinesq equations, which have been extensively studied for their physical significance as well as mathematical importance in recent years (see, e.g., [1-4]). For 2D Boussinesq equations, the global regularity issue has been settled in the affirmative under various degrees of viscosity: with full viscosity $v>0$ and $\kappa>0$, partial viscosity $\nu>0$ and $\kappa=0$, or $v=0$ and $\kappa>0$ for anisotropic models [5-10], and with fractional Laplacian dissipation (see [11-15] and the references therein). For the 3D Boussinesq system and the inviscid case in 2D, we can only obtain the local well-posedness result or the global regularity result with respect to small initial data, see [16-21] etc.

(c) The Author(s) 2019. This article is distributed under the terms of the Creative Commons Attribution 4.0 International License (http://creativecommons.org/licenses/by/4.0/), which permits unrestricted use, distribution, and reproduction in any medium, provided you give appropriate credit to the original author(s) and the source, provide a link to the Creative Commons license, and indicate if changes were made. 
In the meantime, researchers are interested in the stochastic Boussinesq equations by considering that a system in reality is usually affected by external perturbations which in many cases are of great uncertainty or random influence. Ferrario [22] first studied the two-dimensional stochastic Boussinesq system with full viscosity, additive noise only on the velocity field equation, and obtained the existence and uniqueness of its solution and invariant measures for the associated semigroup. Following the work of the deterministic case in [6,7], Pu and Guo [23] studied the stochastic Boussinesq system with partial viscosity with additive noise and obtained the global well-posedness type results. Then, Duan and Millet [24] considered the multiplicative noise case and the large deviations, Brzeźniak and Motyl [25] generalized the existence result (martingale solutions) to the 3D case, and Yamazaki [26] considered the stochastic Boussinesq system with zero dissipation in 2D.

To the best of our knowledge, the problem of the existence and uniqueness of the strong solutions for the 3D stochastic full viscosity Boussinesq equations is still open. In this paper, we prove that the Cauchy problem (1.1) admits a local strong solution under some conditions and obtain a blow-up time. Also, the probability estimate of the lifespan larger than $\delta(0<\delta<1)$ is given in our paper.

Without loss of generality, we take $v=\kappa=1$. We first state the definition of the local strong solutions for the stochastic Boussinesq equations (1.1).

Definition 1.1 Fix stochastic $\mathcal{S}:=\left(\Omega, \mathcal{F}, \mathcal{F}_{t}, \mathbb{P}\right)$. Let $\left(u_{0}, \rho_{0}\right) \in H_{\sigma}^{s} \times H^{s}$ be $\mathcal{F}_{0}$ measurable, $\frac{1}{2}<s \leq 1, U=(u, \rho)$.

(i) A pair $(U, \tau)$ is called a local strong solution of (1.1) if the following conditions are satisfied:

- $U$ is a right continuous progressively measurable process and, for all $0<T<\infty$,

$$
U \in L^{2}\left(\Omega ; L^{\infty}\left([0, T] ; H_{\sigma}^{s} \times H^{s}\right)\right) \cap L^{2}\left(\Omega ; L^{2}\left([0, T] ; H_{\sigma}^{s+1} \times H^{s+1}\right)\right) ;
$$

- $\tau(\omega)$ is a stopping time with respect to $\mathcal{F}_{t}$ such that

$$
\tau(\omega)=\lim _{N \rightarrow \infty} \tau_{N}(\omega) \quad \text { for almost all } \omega
$$

where, for $N=1,2, \ldots$,

$$
\tau_{N}(\omega)=\left\{\begin{array}{l}
\inf \left\{0 \leq t<\infty:\|U(t)\|_{H^{s}}^{2}+\int_{0}^{t}\|\nabla U(s)\|_{H^{s}}^{2} d s \geq N\right\} \\
\infty, \quad \text { if the above set }\{\cdot\} \text { is empty; }
\end{array}\right.
$$

- $U(t, x) \in C\left([0, \tau(\omega)) ; H_{\sigma}^{s} \times H^{s}\right)$ for almost all $\omega \in \Omega$, and the following holds $\mathbb{P}$-a.s.:

$$
\begin{aligned}
\left\langle u\left(t \wedge \tau_{N}\right), \phi\right\rangle-\left\langle u_{0}, \phi\right\rangle= & \int_{0}^{t \wedge \tau_{N}}(\langle\nabla u, \nabla \phi\rangle-\langle u \cdot \nabla u, \phi\rangle) d s \\
& +\left\langle\sum_{i=1}^{\infty} \int_{0}^{t \wedge \tau_{N}} \Phi_{1, i} d W_{i}(s), \phi\right\rangle,
\end{aligned}
$$




$$
\begin{aligned}
\left\langle\rho\left(t \wedge \tau_{N}\right), \psi\right\rangle-\left\langle\rho_{0}, \psi\right\rangle= & \int_{0}^{t \wedge \tau_{N}}(\langle\nabla \rho, \nabla \psi\rangle-\langle u \cdot \nabla \rho, \psi\rangle) d s \\
& +\left\langle\sum_{i=1}^{\infty} \int_{0}^{t \wedge \tau_{N}} \Phi_{2, i} d \widetilde{W}_{i}(s), \psi\right\rangle
\end{aligned}
$$

for all $0 \leq t<\infty$ and all $(\phi, \psi) \in H_{\sigma}^{1} \times H^{1}$. Here $\langle\cdot, \cdot\rangle$ is the inner product, and the definition of spaces will be given in Sect. 2 .

(ii) We say the strong solutions are unique if, given any pair $(U, \tau),(\widetilde{U}, \widetilde{\tau})$ of strong solutions,

$$
\mathbb{P}\left(1_{U(0)=\widetilde{U}(0)}(U(t)-\widetilde{U}(t))=0 ; \forall t \in[0, \tau \wedge \widetilde{\tau})\right)=1 .
$$

Let $\Phi_{j}:=\left\{\Phi_{j, i}\right\}_{i=1}^{\infty}(j=1,2)$. Our main results are stated in the following two theorems.

Theorem 1.2 Fix stochastic $\mathcal{S}:=\left(\Omega, \mathcal{F}, \mathcal{F}_{t}, \mathbb{P}\right)$. Let $\left(u_{0}, \rho_{0}\right) \in L^{2}\left(\Omega ; H_{\sigma}^{s} \times H^{s}\right)$ be $\mathcal{F}_{0}$ measurable, $\left(\Phi_{1}, \Phi_{2}\right) \in L^{2}\left(\Omega ; L_{\text {loc }}^{2}\left(\mathbb{R}^{+} ; \mathbb{H}^{s} \times \mathbb{H}^{s}\right)\right)$ is progressively measurable. Then there exists a unique strong solution $(U, \tau)$ to system (1.1).

Moreover, the blow-up time could be weaker. Indeed, we have the following theorem.

Theorem 1.3 Let $\frac{1}{2}<s^{\prime} \leq s$, define

$$
\begin{aligned}
& \zeta_{K}=\left\{\begin{array}{l}
\inf \left\{t \geq 0:\|u(t)\|_{H^{s^{\prime}}}^{2} \geq K\right\}, \\
\infty, \quad \text { if the set }\{\cdot\} \text { is empty, }
\end{array}\right. \\
& \zeta=\lim _{K \rightarrow \infty} \zeta_{K} .
\end{aligned}
$$

Then $\zeta=\tau$ a.s., and we have

$$
\mathbb{P}(\{\zeta>\delta\}) \geq 1-C^{*} \delta^{\frac{2 s^{\prime}-1}{2 s^{\prime}+1}}(\mathcal{A}(\delta)+1),
$$

where

$$
\mathcal{A}(\delta)=\mathbb{E}\left(\left\|u_{0}\right\|_{H^{s^{\prime}}}^{2}+\int_{0}^{\delta}\left\|\Phi_{1}\right\|_{\mathbb{H}^{s^{\prime}}}^{2} d t\right)+\delta \mathbb{E}\left(\|\rho\|_{L^{2}}^{2}+\int_{0}^{\delta}\left\|\Phi_{2}\right\|_{\mathbb{L}^{2}}^{2}\right)
$$

Remark 1.4 In fact, we can consider the more general case that the solution $U \in H_{0}^{s} \times H^{n}$ $\left(\frac{1}{2}<s, \frac{1}{2}<n, s-2 \leq n \leq s\right)$. The proof is similar to ours.

We can construct the strong solution by the contraction mapping principle, cut-off function method, and Cauchy convergence theorem. For the detailed procedure, refer to [27]. Theorem 1.3 can be proved by using the stopping time and energy estimates.

The rest of the paper is organized as follows. We shall introduce some analysis tools in Sobolev spaces and some basic theory of stochastic analysis in Sect. 2. In Sect. 3, we shall prove the local existence of the strong solution, and Theorem 1.3 will be proved in the last section. 


\section{Preliminaries}

Let $H^{m}=W^{m, 2}\left(\mathbb{R}^{3}\right)$ be the usual Sobolev space. We use the same notation $H^{m}$ for $H^{m}\left(\mathbb{R}^{3} ; \mathbb{R}^{3}\right)$ also. The symbols $\langle\cdot\rangle_{H^{m}}$ and $\|\cdot\|_{H^{m}}$ represent the inner product and the norm of $H^{m}$, respectively. We introduce the function space

$$
H_{\sigma}^{m}=\left\{f \in H^{m} ; \nabla \cdot f=0\right\}
$$

The symbol $\mathbf{P}$ denotes the projection $H^{m} \rightarrow H_{\sigma}^{m}$. We recall the following inequality which will be used later: for all $v \in H^{s^{\prime}}, u \in H^{\frac{3}{2}+s-s^{\prime}}$, and $w \in H^{s+1}, \frac{1}{2}<s^{\prime} \leq s \leq 1$, we have

$$
\left|\langle v \cdot \nabla u, w\rangle_{H^{s}}\right| \leq C\|v\|_{H^{s^{\prime}}}\|\nabla u\|_{H^{\frac{1}{2}+s-s^{\prime}}}\|w\|_{H^{s+1}}
$$

since $H^{s^{\prime}} \subset L^{p}, H^{\frac{1}{2}+s-s^{\prime}} \subset L^{q}, H^{1-s} \subset L^{r}, 1 / p+1 / q+1 / r=1$. By the interpolation inequality, we have

$$
\|\nabla u\|_{H^{\frac{1}{2}+s-s^{\prime}}} \leq C\|u\|_{H^{s}}^{s^{\prime}-\frac{1}{2}}\|u\|_{H^{s+1}}^{\frac{3}{2}-s^{\prime}} .
$$

We define the space for the white noise. Let $\Phi:=\left\{\Phi_{i}\right\}_{i=1}^{\infty}$, suppose $m \geq 0$, define

$$
\mathbb{H}^{m}:=\left\{\Phi \mid \forall i, \Phi_{i} \in H^{m} \text { and } \sum_{i=1}^{\infty}\left\|\Phi_{i}\right\|_{H^{m}}^{2}<\infty\right\},
$$

with the norm

$$
\|\Phi\|_{\mathbb{H}^{m}}^{2}:=\sum_{i=1}^{\infty}\left\|\Phi_{i}\right\|_{H^{m}}^{2}
$$

When $s=0$, we also denote $\mathbb{L}^{2}$ by $\mathbb{H}^{0}$.

Now, we recall some basic theory of stochastic analysis. For details, we refer the reader to $[28-31]$ and the references therein.

Lemma 2.1 Suppose that $M_{t}=\left(M_{t}^{1}, M_{t}^{2}, \ldots, M_{t}^{n}\right)$ is a vector of continuous local martingales, that is, $\left(M_{t}^{i}, \mathcal{F}_{t}\right)$ is a local martingale for each $i=1,2, \ldots, n$ and $t \in \mathbb{R}^{+}$. Let $A_{t}=$ $\left(A_{t}^{1}, A_{t}^{2}, \ldots, A_{t}^{n}\right)$ be a vector of continuous process adapted to the same filtration such that the total variation of $A_{t}^{i}$ on each finite interval is bounded almost surely, and $A_{0}^{i}=0$ almost surely. Let $X_{t}=\left(X_{t}^{1}, X_{t}^{2}, \ldots, X_{t}^{n}\right)$ be a vector of adapted processes such that $X_{t}=X_{0}+M_{t}+A_{t}$, and let $f \in C^{1,2}\left(\mathbb{R}^{+} \times \mathbb{R}^{n}\right)$. Then, for any $t \geq 0$, the equality

$$
\begin{aligned}
f\left(t, X_{t}\right)= & f\left(0, X_{0}\right)+\sum_{i=1}^{n} \int_{0}^{t} \frac{\partial}{\partial x_{i}} f\left(s, X_{s}\right) d M_{s}^{i}+\sum_{i=1}^{n} \int_{0}^{t} \frac{\partial}{\partial x_{i}} f\left(s, X_{s}\right) d A_{s}^{i} \\
& +\frac{1}{2} \sum_{i=1}^{n} \int_{0}^{t} \frac{\partial^{2}}{\partial_{x_{i}} \partial_{x_{j}}} f\left(s, X_{s}\right) d\left\langle M^{i}, M^{j}\right\rangle_{s}+\int_{0}^{t} \frac{\partial}{\partial s} f\left(s, X_{s}\right) d s
\end{aligned}
$$

holds almost surely. Here $\langle\cdot, \cdot\rangle_{t}$ is the cross variation process defined by $\langle X, Y\rangle_{t}=\frac{1}{4}\{\langle X+$ $\left.Y\rangle_{t}-\langle X-Y\rangle_{t}\right\}$, and $\langle X\rangle_{t}$ denotes the quadratic variation of $X$ on $[0, t]$. 
Lemma 2.2 (Burkholder-Davis-Gundy inequality) Let $T>0$ and $\left\{X_{t}\right\}_{0 \leq t \leq T}$ be a continuous local martingale such that $X_{0}=0$. For every $0<p<\infty$, there exist universal constants $c_{p}$ and $C_{p}$, independent of $T$ and $X_{t}$, such that

$$
c_{p} \mathbb{E}\left(\langle X\rangle_{T}^{\frac{p}{2}}\right) \leq \mathbb{E}\left(\max _{0 \leq t \leq T}\left|X_{s}\right|^{p}\right) \leq C_{p} \mathbb{E}\left(\langle X\rangle_{T}^{\frac{p}{2}}\right) .
$$

\section{The local existence of local strong solution}

We first give the key estimate to prove the local existence and uniqueness of the strong solution. Fix $N>0$ to be determined, choose a $C^{\infty}$-smooth nonincreasing function $\varphi_{N}$ : $[0, \infty) \rightarrow[0,1]$ such that

$$
\varphi_{N}(x)= \begin{cases}1, & \text { for }|x|<N \\ 0, & \text { for }|x|>N+1\end{cases}
$$

and

$$
\varphi_{N}^{u, \rho}=\varphi_{N}\left(\|u\|_{H^{s}}+\|\rho\|_{H^{s}}+\left(\int_{0}^{t}\left(\|\nabla u\|_{H^{s}}^{2}+\|\nabla \rho\|_{H^{s}}^{2}\right) d s\right)^{1 / 2}\right) .
$$

We consider the following Cauchy problem:

$$
\left\{\begin{array}{l}
d u^{j}+\left(\varphi_{N}^{u^{j}, \rho^{j}} \mathbf{P}\left(u^{j} \cdot \nabla u^{j}\right)-\Delta u^{j}\right) d t=\mathbf{P}\left(\rho^{j} e_{3}\right) d t+\sum_{i=1}^{\infty} \mathbf{P} \Phi_{1, i}^{j} d W_{i}, \\
d \rho^{j}+\left(\varphi_{N}^{u^{j}, \rho^{j}}\left(u^{j} \cdot \nabla \rho^{j}\right)-\Delta \rho^{j}\right) d t=\sum_{i=1}^{\infty} \Phi_{2, i}^{j} d \widetilde{W}_{i}, \\
\left.\left(u^{j}, \rho^{j}\right)\right|_{t=0}=\left(u_{0}^{j}, \rho_{0}^{j}\right),
\end{array}\right.
$$

for $j=1,2$. By Itô's formula in $H^{s}$ and the equations of $\left(u^{j}, \rho^{j}\right)$, we have

$$
\begin{aligned}
d \| u^{1} & -u^{2}\left\|_{H^{s}}^{2}+2\right\| \nabla u^{1}-\nabla u^{2} \|_{H^{s}}^{2} d t \\
= & -2\left\langle\varphi_{N}^{u^{1}, \rho^{1}}\left(u^{1} \cdot \nabla u^{1}\right)-\varphi_{N}^{u^{2}, \rho^{2}}\left(u^{2} \cdot \nabla u^{2}\right), u^{1}-u^{2}\right\rangle_{H^{s}} d t \\
& +\left\|\Phi_{1}^{1}-\Phi_{1}^{2}\right\|_{H^{s}}^{2} d t+2\left\langle\Phi_{1, i}^{1}-\Phi_{1, i}^{2}, u^{1}-u^{2}\right\rangle_{H^{s}} d W_{i} \\
& +2\left\langle\left(\rho^{1}-\rho^{2}\right) e_{3}, u^{1}-u^{2}\right\rangle_{H^{s}} d t,
\end{aligned}
$$

and

$$
\begin{aligned}
d \| \rho^{1}- & \rho^{2}\left\|_{H^{s}}^{2}+2\right\| \nabla \rho^{1}-\nabla \rho^{2} \|_{H^{s}}^{2} d t \\
= & -2\left\langle\varphi_{N}^{u^{1}, \rho^{1}}\left(u^{1} \cdot \nabla \rho^{1}\right)-\varphi_{N}^{u^{2}, \rho^{2}}\left(u^{2} \cdot \nabla \rho^{2}\right), \rho^{1}-\rho^{2}\right\rangle_{H^{s}} d t \\
& +\left\|\Phi_{1}^{2}-\Phi_{2}^{2}\right\|_{\mathbb{H}^{s}}^{s} d t+2\left\langle\Phi_{i, 1}^{2}-\Phi_{i, 2}^{2}, \rho^{1}-\rho^{2}\right\rangle_{H^{s}} d \widetilde{W}_{i} .
\end{aligned}
$$

We have the following proposition.

Proposition 3.1 For any $T>0$, we have

$$
\begin{gathered}
\mathbb{E}\left(\sup _{0 \leq t \leq T}\left(\|\bar{u}(t)\|_{H^{s}}^{2}+\|\bar{\rho}(t)\|_{H^{s}}^{2}\right)+\int_{0}^{T}\left(\|\nabla \bar{u}\|_{H^{s}}^{2}+\|\nabla \bar{\rho}\|_{H^{s}}^{2}\right) d t\right) \\
\leq e^{C_{N, T}} \mathbb{E}\left(\left\|\bar{u}_{0}\right\|_{H^{s}}^{2}+\left\|\bar{\rho}_{0}\right\|_{H^{s}}^{2}+\int_{0}^{T}\left(\left\|\bar{\Phi}_{1}\right\|_{\mathbb{H}^{s}}^{2}+\left\|\bar{\Phi}_{2}\right\|_{\mathbb{H}^{s}}^{2} d t\right),\right.
\end{gathered}
$$

where $\bar{u}=u_{1}-u_{2}, \bar{\rho}=\rho_{1}-\rho_{2}$, and $\bar{\Phi}_{i}=\Phi_{i}^{1}-\Phi_{i}^{2}(i=1,2)$. 
Proof Set

$$
\begin{aligned}
& Q_{1}(t)=\left|\left\langle\varphi_{N}^{u^{1}, \rho^{1}}\left(u^{1} \cdot \nabla u^{1}\right)-\varphi_{N}^{u^{2}, \rho^{2}}\left(u^{2} \cdot \nabla u^{2}\right), u^{1}-u^{2}\right\rangle_{H^{s}}\right|, \\
& Q_{2}(t)=\left|\left\langle\varphi_{N}^{u^{1}, \rho^{1}}\left(u^{1} \cdot \nabla \rho^{1}\right)-\varphi_{N}^{u^{2}, \rho^{2}}\left(u^{1} \cdot \nabla \rho^{2}\right), \rho^{1}-\rho^{2}\right\rangle_{H^{s}}\right| .
\end{aligned}
$$

We consider three cases:

Case 1: $\varphi_{N}^{u^{1}, \rho^{1}}>0, \varphi_{N}^{u^{2}, \rho^{2}}>0$ :

$$
\begin{aligned}
Q_{1}(t) \leq & \left|\varphi_{N}^{u^{1}, \rho^{1}}-\varphi_{N}^{u^{2}, \rho^{2}}\right|\left|\left\langle u^{1} \cdot \nabla u^{1}, u^{1}-u^{2}\right\rangle_{H^{s}}\right| \\
& +\left|\left\langle\left(u^{1}-u^{2}\right) \cdot \nabla u^{1}, u^{1}-u^{2}\right\rangle_{H^{s}}\right| \\
& +\left|\left\langle u^{2} \cdot \nabla\left(u^{1}-u^{2}\right), u^{1}-u^{2}\right\rangle_{H^{s}}\right| \\
:= & I_{1}+I_{2}+I_{3}, \\
Q_{2}(t) \leq & \left|\varphi_{N}^{u^{1}, \rho^{1}}-\varphi_{N}^{u^{2}, \rho^{2}}\right|\left|\left\langle u^{1} \cdot \nabla \rho^{1}, \rho^{1}-\rho^{2}\right\rangle_{H^{s}}\right| \\
& +\left|\left\langle\left(u^{1}-u^{2}\right) \cdot \nabla \rho^{1}, \rho^{1}-\rho^{2}\right\rangle_{H^{s}}\right| \\
& +\left|\left\langle u^{2} \cdot \nabla\left(\rho^{1}-\rho^{2}\right), \rho^{1}-\rho^{2}\right\rangle_{H^{s}}\right| \\
:= & J_{1}+J_{2}+J_{3} .
\end{aligned}
$$

Case 2: $\varphi_{N}^{u^{1}, \rho^{1}}>0, \varphi_{N}^{u^{2}, \rho^{2}}=0$ :

$$
\begin{aligned}
& Q_{1}(t) \leq\left|\varphi_{N}^{u^{1}, \rho^{1}}-\varphi_{N}^{u^{2}, \rho^{2}}\right|\left|\left\langle u^{1} \cdot \nabla u^{1}, u^{1}-u^{2}\right\rangle_{H^{s}}\right| \\
& Q_{2}(t) \leq\left|\varphi_{N}^{u^{1}, \rho^{1}}-\varphi_{N}^{u^{2}, \rho^{2}}\right|\left|\left\langle u^{1} \cdot \nabla \rho^{1}, \rho^{1}-\rho^{2}\right\rangle_{H^{s}}\right| .
\end{aligned}
$$

Case 3: $\varphi_{N}^{u^{1}, \rho^{1}}=0, \varphi_{N}^{u^{2}, \rho^{2}}>0$ :

$$
\begin{aligned}
& Q_{1}(t) \leq\left|\varphi_{N}^{u^{1}, \rho^{1}}-\varphi_{N}^{u^{2}, \rho^{2}}\right|\left|\left\langle u^{2} \cdot \nabla u^{2}, u^{1}-u^{2}\right\rangle_{H^{s}}\right| \\
& Q_{2}(t) \leq\left|\varphi_{N}^{u^{1}, \rho^{1}}-\varphi_{N}^{u^{2}, \rho^{2}}\right|\left|\left\langle u^{2} \cdot \nabla \rho^{2}, \rho^{1}-\rho^{2}\right\rangle_{H^{s}}\right| .
\end{aligned}
$$

Denote

$$
\begin{aligned}
Y(t)= & \sup _{0 \leq t^{\prime} \leq t}\left(\left\|u^{1}\left(t^{\prime}\right)-u^{2}\left(t^{\prime}\right)\right\|_{H^{s}}^{2}+\left\|\rho^{1}\left(t^{\prime}\right)-\rho^{2}\left(t^{\prime}\right)\right\|_{H^{s}}^{2}\right) \\
& +\int_{0}^{t}\left(\left\|\nabla u^{1}-\nabla u^{2}\right\|_{H^{s}}+\left\|\nabla \rho_{1}-\nabla \rho_{2}\right\|_{H^{s}}^{2}\right) d t^{\prime},
\end{aligned}
$$

we shall estimate $Q_{1}(t)$ and $Q_{2}(t)$. For $Q_{1}(t)$, by taking $s^{\prime}=s$ in (2.1) and (2.2), we have

$$
\begin{aligned}
I_{1} & \leq C Y(t)^{1 / 2}\left\|u^{1}-u^{2}\right\|_{H^{s+1}}\left\|u^{1}\right\|_{H^{s}}\left\|\nabla u^{1}\right\|_{H^{\frac{1}{2}}} \\
& \leq C Y(t)^{1 / 2}\left\|u^{1}-u^{2}\right\|_{H^{s+1}}\left\|u^{1}\right\|_{H^{s}}^{s+\frac{1}{2}}\left\|u^{1}\right\|_{H^{s+1}}^{\frac{3}{2}-s} \\
& \leq \frac{1}{4}\left\|u^{1}-u^{2}\right\|_{H^{s+1}}^{2}+C Y(t)\left\|u^{1}\right\|_{H^{s}}^{2 s+1}\left\|u^{1}\right\|_{H^{s+1}}^{3-2 s},
\end{aligned}
$$




$$
\begin{aligned}
I_{2} & \leq C\left\|u^{1}-u^{2}\right\|_{H^{s}}\left\|\nabla u^{1}\right\|_{H^{\frac{1}{2}}}\left\|u^{1}-u^{2}\right\|_{H^{s+1}} \\
& \leq C\left\|u^{1}-u^{2}\right\|_{H^{s+1}}\left\|u_{1}\right\|_{H^{s}}^{s+\frac{1}{2}}\left\|u^{1}\right\|_{H^{s+1}}^{\frac{3}{2}-s}\left\|u^{1}-u^{2}\right\|_{H^{s+1}} \\
& \leq \frac{1}{4}\left\|u^{1}-u^{2}\right\|_{H^{s+1}}^{2}+C\left\|u_{1}\right\|_{H^{s}}^{2 s+1}\left\|u^{1}\right\|_{H^{s+1}}^{3-2 s}\left\|u_{1}-u_{2}\right\|_{H^{s}}^{2}, \\
& \leq \frac{1}{4}\left\|u^{1}-u^{2}\right\|_{H^{s+1}}^{2}+C Y(t)\left\|u_{1}\right\|_{H^{s}}^{2 s+1}\left\|u^{1}\right\|_{H^{s+1}}^{3-2 s}, \\
I_{3} & \leq C\left\|u^{2}\right\|_{H^{s}}\left\|\nabla\left(u^{1}-u^{2}\right)\right\|_{H^{\frac{1}{2}}}\left\|u^{1}-u^{2}\right\|_{H^{s+1}} \\
& \leq C\left\|u^{2}\right\|_{H^{s}}\left\|u^{1}-u^{2}\right\|_{H^{s}}^{s-\frac{1}{2}}\left\|u^{1}-u^{2}\right\|_{H^{s+1}}^{\frac{5}{2}-s} \\
& \leq \frac{1}{4}\left\|u^{1}-u^{2}\right\|_{H^{s+1}}^{2}+C\left\|u^{1}-u^{2}\right\|_{H^{s}}^{2}\left\|u^{2}\right\|_{H^{s}}^{\frac{4}{2 s-1}} \\
& \leq \frac{1}{4}\left\|u^{1}-u^{2}\right\|_{H^{s+1}}^{2}+C Y(t)\left\|u^{2}\right\|_{H^{s}}^{\frac{4}{2 s-1}} .
\end{aligned}
$$

Hence, it holds that

$$
Q_{1}(t) \leq \frac{3}{4}\left\|\nabla u^{1}-\nabla u^{2}\right\|_{H^{s}}^{2}+R_{1}(t) Y(t)
$$

where

$$
\int_{0}^{T} R_{1}(t) d t \leq(N+1)^{\frac{4}{2 s-1}}+(N+1) T^{s-\frac{1}{2}}
$$

according to the definition of $\varphi_{N}^{u}$. Similarly, we have

$$
\begin{aligned}
& J_{1} \leq \frac{1}{4}\left\|\rho^{1}-\rho^{2}\right\|_{H^{s+1}}^{2}+C Y(t)\left\|\rho^{1}\right\|_{H^{s}}^{2 s+1}\left\|\rho^{1}\right\|_{H^{s+1}}^{3-2 s}, \\
& J_{2} \leq \frac{1}{4}\left\|\rho^{1}-\rho^{2}\right\|_{H^{s+1}}^{2}+C Y(t)\left\|\rho_{1}\right\|_{H^{s}}^{2 s+1}\left\|u^{1}\right\|_{H^{s+1}}^{3-2 s}, \\
& J_{3} \leq \frac{1}{4}\left\|\rho^{1}-\rho^{2}\right\|_{H^{s+1}}^{2}+C Y(t)\left\|u^{2}\right\|_{H^{s}}^{\frac{4}{2 s-1}} .
\end{aligned}
$$

Therefore

$$
Q_{2}(t) \leq \frac{3}{4}\left\|\nabla \rho^{1}-\nabla \rho^{2}\right\|_{H^{1}}^{2}+R_{2}(t) Y(t),
$$

and

$$
\int_{0}^{T} R_{1}(t) d t \leq(N+1)^{\frac{4}{2 s-1}}+(N+1) T^{s-\frac{1}{2}} .
$$

In addition,

$$
\begin{aligned}
\left|\left\langle\left(\rho^{1}-\rho^{2}\right) e_{3}, u^{1}-u^{2}\right\rangle_{H^{s}}\right| & \leq C\left(\left\|\rho^{1}-\rho^{2}\right\|_{H^{s}}^{2}+\left\|u^{1}-u^{2}\right\|_{H^{s}}^{2}\right) \\
& \leq C Y(t) .
\end{aligned}
$$


Introducing a stopping time:

$$
\sigma_{L}=\left\{\begin{array}{l}
\inf \{0 \leq t \leq T: Y(t)>L\} \\
T, \quad \text { if the set }\{\cdot\} \text { is empty }
\end{array}\right.
$$

Therefore, combining (3.2)-(3.19) and recalling the classical Grönwall inequality, we get

$$
\begin{aligned}
Y\left(T \wedge \sigma_{L}\right) \leq & e^{C_{N, T}}\left(\left\|u_{0}^{1}-u_{0}^{2}\right\|_{H^{s}}^{2}+\left\|\rho_{0}^{1}-\rho_{0}^{2}\right\|_{H^{s}}^{2}\right) \\
& +e^{C_{N, T}} \sup _{t \in\left[0, T \wedge \sigma_{L}\right]}\left|\sum_{i=1}^{\infty} \int_{0}^{t}\left\langle u^{1}-u^{2}, \Phi_{i, 1}^{1}-\Phi_{i, 1}^{2}\right\rangle_{H^{s}} d W_{i}\right| \\
& +e^{C_{N, T}} \sup _{t \in\left[0, T \wedge \sigma_{L}\right]}\left|\sum_{i=1}^{\infty} \int_{0}^{t}\left\langle\rho^{1}-\rho^{2}, \Phi_{i, 2}^{1}-\Phi_{i, 2}^{2}\right\rangle_{H^{s}} d \widetilde{W}_{i}\right| \\
& +e^{C_{N, T}} \int_{0}^{T \wedge \sigma_{L}}\left(\left\|\Phi_{1}^{1}-\Phi_{1}^{2}\right\|_{\mathbb{H}^{s}}^{2}+\left\|\Phi_{2}^{1}-\Phi_{2}^{2}\right\|_{\mathbb{H}^{s}}^{2}\right) d t .
\end{aligned}
$$

Furthermore, by the Burkholder-Davis-Gundy inequality (2.4), one has

$$
\begin{gathered}
e^{C_{N, T}} \mathbb{E}\left(\sup _{t \in\left[0, T \wedge \sigma_{L}\right]}\left|\sum_{i=1}^{\infty} \int_{0}^{t}\left\langle u^{1}-u^{2}, \Phi_{i, 1}^{1}-\Phi_{i, 1}^{2}\right\rangle_{H^{s}} d W_{i}\right|\right) \\
\leq e^{C_{N, T}} \mathbb{E}\left(\int_{0}^{T \wedge \sigma_{L}} Y(t)\left\|\Phi_{i, 1}^{1}-\Phi_{i, 1}^{2}\right\|_{\mathbb{H}^{s}}^{2} d t\right)^{1 / 2} \\
\leq \frac{1}{4} \mathbb{E} Y\left(T \wedge \sigma_{L}\right)+e^{C_{N, T}} \mathbb{E} \int_{0}^{T}\left\|\Phi_{i, 1}^{1}-\Phi_{i, 1}^{2}\right\|_{\mathbb{H}^{s}}^{2} d t
\end{gathered}
$$

and

$$
\begin{aligned}
& e^{C_{N, T}} \mathbb{E}\left(\sup _{t \in\left[0, T \wedge \sigma_{L}\right]}\left|\sum_{i=1}^{\infty} \int_{0}^{t}\left\langle\rho^{1}-\rho^{2}, \Phi_{i, 2}^{1}-\Phi_{i, 2}^{2}\right\rangle_{H^{s}} d \widetilde{W}_{i}\right|\right) \\
& \leq e^{C_{N, T}} \mathbb{E}\left(\int_{0}^{T \wedge \sigma_{L}} Y(t)\left\|\Phi_{i, 2}^{1}-\Phi_{i, 2}^{2}\right\|_{\mathbb{H}^{s}}^{2} d t\right)^{1 / 2} \\
& \leq \frac{1}{4} \mathbb{E} Y\left(T \wedge \sigma_{L}\right)+e^{C_{N, T}} \mathbb{E} \int_{0}^{T}\left\|\Phi_{i, 2}^{1}-\Phi_{i, 2}^{2}\right\|_{\mathbb{H}^{s}}^{2} d t
\end{aligned}
$$

Taking the mathematical expectation in (3.20), (3.21), and (3.22) implies

$$
\begin{aligned}
\mathbb{E} Y(T) \leq & e^{C_{N, T}} \mathbb{E}\left(\left\|u_{0}^{1}-u_{0}^{2}\right\|_{H^{s}}^{2}+\left\|\rho_{0}^{1}-\rho_{0}^{2}\right\|_{H^{s}}^{2}\right) \\
& +e^{C_{N, T}} \mathbb{E} \int_{0}^{T}\left(\left\|\Phi_{1}^{1}-\Phi_{1}^{2}\right\|_{\mathbb{H}^{s}}^{2}+\left\|\Phi_{2}^{1}-\Phi_{2}^{2}\right\|_{\mathbb{H}^{s}}^{2}\right) d t .
\end{aligned}
$$

Hence, we get the key estimate for proving the existence of strong solution. Now, we give the sketch for the proof of the existence and uniqueness of strong solution. 
1. Let $v_{\varepsilon}$ be the Friedrichs mollifier. By the contraction mapping principle, we can show that the approximate mollified equation

$$
\begin{aligned}
& d u_{\varepsilon}+\left(\varphi_{N}^{u_{\varepsilon_{i}}, \rho_{\varepsilon_{i}}} \mathbf{P}\left(u_{\varepsilon} \cdot \nabla u_{\varepsilon}\right)-\Delta u_{\varepsilon}\right) d t=\mathbf{P} \rho_{\varepsilon} e_{3} d t+\sum_{i=1}^{\infty} \mathbf{P} \Phi_{1, i} * v_{\varepsilon} d W_{i}, \\
& d \rho_{\varepsilon}+\left(\varphi_{N}^{u_{\varepsilon_{i}}, \rho_{\varepsilon_{i}}} u_{\varepsilon} \cdot \nabla \rho_{\varepsilon}-\Delta \rho_{\varepsilon}\right) d t=\sum_{i=1}^{\infty} \Phi_{2, i} * v_{\varepsilon} d \widetilde{W}_{i}, \\
& \left.\left(u_{\varepsilon}, \rho_{\varepsilon}\right)\right|_{t=0}=\left(u_{0} * v_{\varepsilon}, \rho_{0} * v_{\varepsilon}\right),
\end{aligned}
$$

admits a unique global strong solution.

2. It follows from (3.23) that $\left\{U_{\varepsilon_{i}}\right\}$ is a Cauchy sequence in

$$
L^{2}\left(\Omega ; C\left([0, T] ; H_{\sigma}^{s} \times H^{s}\right)\right) \cap L^{2}\left(\Omega ; L^{2}\left(0, T ; H_{\sigma}^{s+1} \times H^{s+1}\right)\right) .
$$

Let $U$ be the limit. We can extract a subsequence still denoted by $U_{\varepsilon_{i}}$ such that

$$
U_{\varepsilon_{i}}(\omega) \rightarrow U(\omega) \quad \text { in } C\left([0, T] ; H_{\sigma}^{s} \times H^{s}\right) \cap L^{2}\left(0, T ; H_{\sigma}^{s+1} \times H^{s+1}\right) \quad \mathbb{P} \text {-a.s. }
$$

Then $\varphi_{N}^{u_{\varepsilon_{i}}, \rho_{\varepsilon_{i}}} \rightarrow \varphi_{N}^{u, \rho}$. Hence $U$ is the unique solution of the modified equation

$$
\begin{aligned}
& d u+\left(\varphi_{N}^{u, \rho} \mathbf{P}(u \cdot \nabla u)-\Delta u\right) d t=\mathbf{P} \rho e_{3} d t+\sum_{i=1}^{\infty} \mathbf{P} \Phi_{1, i} d W_{i} \\
& d \rho+\left(\varphi_{N}^{u, \rho} u \cdot \nabla \rho-\Delta \rho\right) d t=\sum_{i=1}^{\infty} \Phi_{2, i} d \tilde{W}_{i}, \\
& \left.(u, \rho)\right|_{t=0}=\left(u_{0}, \rho_{0}\right) .
\end{aligned}
$$

3. Define the stopping time, and drop the cut-off function by the uniqueness of $U$, we can get the local strong solution of (1.1). If there are two solutions $U$ defined for $[0, \tau)$ and $\widetilde{U}$ defined for $[0, \widetilde{\tau})$, then by inequality (3.4), for any $N \geq 1$,

$$
U=\widetilde{U} \quad \text { on }\left[0, \tau_{N} \wedge \widetilde{\tau}_{N}\right]
$$

If $\tilde{\tau}_{N}<\tau_{N}$, then $\left\|U\left(\tau_{N}\right)\right\|_{H^{s}}^{2} \geq N$, which contradicts the definition of $\tau_{N}$ in (1.2). Hence $\tau_{N} \leq \tilde{\tau}_{N}$. Similarly, we have $\widetilde{\tau}_{N} \leq \tau_{N}$, thus $\tilde{\tau}_{N}=\tau_{N}$. Therefore, we obtain the uniqueness of solutions.

\section{Proof of Theorem 1.3}

\subsection{A new blow-up time}

We introduce another stopping time as follows:

$$
\widetilde{\zeta}_{K}=\left\{\begin{array}{l}
\inf \left\{t \geq 0:\|u(t \wedge \tau)\|_{H^{s^{\prime}}}^{2} \geq K\right\} \\
\tau, \quad \text { if the set }\{\cdot\} \text { is empty. }
\end{array}\right.
$$

We have the following proposition. 
Proposition 4.1 For any $K>0, \widetilde{\zeta}_{K}<\tau$ almost surely on the set $\{\tau<\infty\}$

Proof Define $S_{k}^{N}=\left\{\tau_{N} \leq \widetilde{\zeta}_{K}\right\} \cap\left\{\tau_{N} \leq k\right\}$ for $k \geq 1$ and $N \geq 1$. According to Itô's formula and the equation of $(u, \rho)$, we have

$$
\begin{aligned}
d\left(\|u\|_{H^{s}}^{2}+\|\rho\|_{H^{s}}^{2}\right)+2\left(\|\nabla u\|_{H^{s}}^{2}+\|\nabla \rho\|_{H^{s}}^{2}\right) d t & =\left(-2\langle u \cdot \nabla u, u\rangle_{H^{s}}-2\langle u \cdot \nabla \rho, \rho\rangle_{H^{s}}+\left\langle\rho e_{3}, u\right\rangle_{H^{s}}\right) d t \\
& \quad+2 \sum_{i=1}^{\infty}\left(\left\langle\Phi_{1, i}, u\right\rangle_{H^{s}} d W_{i}+\left\langle\Phi_{2, i}, \rho\right\rangle_{H^{s}} d \widetilde{W}_{i}\right)+\left(\left\|\mathbf{P} \Phi_{1}\right\|_{\mathbb{H}^{s}}^{2}+\left\|\Phi_{2}\right\|_{\mathbb{H}^{s}}^{2}\right) d t .
\end{aligned}
$$

By (2.1) and (2.2), Young's inequality, and Hölder's inequality, we bound

$$
\begin{aligned}
\left|\langle u \cdot \nabla u, u\rangle_{H^{s}}\right| & \leq\|u\|_{H^{s^{\prime}}}\|u\|_{H^{\frac{3}{2}+s-s^{\prime}}}\|u\|_{H^{s+1}} \\
& \leq\|u\|_{H^{s^{\prime}}}\|u\|_{H^{s}}^{s^{\prime}-\frac{1}{2}}\|u\|_{H^{s+1}}^{\frac{5}{2}-s^{\prime}} \\
& \leq \frac{1}{2}\|\nabla u\|_{H^{s}}^{2}+C\|u\|_{H^{s}}^{2}\left(1+\|u\|_{H^{s^{\prime}}}^{\frac{4}{2 s^{\prime}-1}}\right), \\
\left|\langle u \cdot \nabla \rho, \rho\rangle_{H^{s}}\right| & \leq\|u\|_{H^{s^{\prime}}}\|\rho\|_{H^{\frac{3}{2}+s-s^{\prime}}}\|\rho\|_{H^{s+1}} \\
& \leq\|u\|_{H^{s^{\prime}}}\|\rho\|_{H^{s^{s}}}^{s^{\prime}-\frac{1}{2}}\|\rho\|_{H^{s+1}}^{\frac{5}{2}-s^{\prime}} \\
& \leq \frac{1}{2}\|\nabla \rho\|_{H^{s}}^{2}+C\|\rho\|_{H^{s}}^{2}\left(1+\|u\|_{H^{s^{\prime}}}^{\frac{4}{2 s^{\prime}-1}}\right),
\end{aligned}
$$

and

$$
\begin{aligned}
\left|\left\langle\rho e_{3}, u\right\rangle_{H^{s}}\right| & \leq C\|\rho\|_{H^{s}}\|u\|_{H^{s}} \\
& \leq C\left(\|\rho\|_{H^{s}}^{2}+\|u\|_{H^{s}}^{2}\right) .
\end{aligned}
$$

By Grönwall's inequality, we obtain

$$
\begin{aligned}
& \sup _{t \in\left[0, k \wedge \tau_{N} \wedge \widetilde{\zeta}_{K}\right]}\left(\|u\|_{H^{s}}^{2}+\|\rho\|_{H^{s}}^{2}\right)+\int_{0}^{k \wedge \tau_{N} \wedge \widetilde{\zeta}_{K}}\left(\|\nabla u\|_{H^{s}}^{2}+\|\nabla \rho\|_{H^{s}}^{2}\right) d s \\
& \leq C\left(\left\|u_{0}\right\|_{H^{s}}^{2}+\left\|\rho_{0}\right\|_{H^{s}}^{2}+\int_{0}^{k \wedge \tau_{N} \wedge \widetilde{\zeta}_{K}}\left(\left\|\Phi_{1}\right\|_{\mathbb{H}^{s}}^{2}+\left\|\Phi_{2}\right\|_{\mathbb{H}^{s}}^{2}\right) d t\right. \\
& \left.\quad+\sup _{t \in\left[0, k \wedge \tau_{N} \wedge \widetilde{\zeta}_{K}\right]} \sum_{i=1}^{\infty}\left|\int_{0}^{t}\left\langle\Phi_{1, i}, u\right\rangle_{H^{s}} d W_{i}+\int_{0}^{t}\left\langle\Phi_{2, i}, \rho\right\rangle_{H^{s}} d \widetilde{W}_{i}\right|\right) \\
& \quad \times \exp \left(C \int_{0}^{k \wedge \tau_{N} \wedge \widetilde{\zeta}_{K}}\left(1+\|u\|_{H^{s^{s}}}^{\frac{4}{2 s^{\prime}-1}}\right) d s\right) .
\end{aligned}
$$

According to the definition of $\widetilde{\zeta}_{K}$, we have

$$
\int_{0}^{k \wedge \tau_{N} \wedge \widetilde{\zeta} K}\left(1+\|u\|_{H^{s^{\prime}}}^{\frac{4}{2 s^{\prime}-1}}\right) d s \leq\left(1+K^{\frac{2}{2 s^{\prime}-1}}\right) k \quad \text { a.s. }
$$


Furthermore, by the Burkholder-Davis-Gundy inequality (2.4), one has

$$
\begin{aligned}
& e^{C_{K, k} \mathbb{E}} \sup _{t \in\left[0, k \wedge \tau_{N} \wedge \widetilde{\zeta}_{K}\right]} \sum_{i=1}^{\infty}\left|\int_{0}^{t}\left\langle\Phi_{1, i}, u\right\rangle_{H^{s}} d W_{i}+\int_{0}^{t}\left\langle\Phi_{2, i}, \rho\right\rangle_{H^{s}} d \widetilde{W}_{i}\right| \\
& \leq e^{C_{K, k} \mathbb{E}}\left(\int_{0}^{k \wedge \tau_{N} \wedge \tilde{\zeta}_{K}}\left(\|u\|_{H^{s}}^{2}\left\|\Phi_{1}\right\|_{\mathbb{H}^{s}}^{2}+\|\rho\|_{H^{s}}^{2}\left\|\Phi_{2}\right\|_{\mathbb{H}^{s}}^{2}\right) d s\right)^{1 / 2} \\
& \leq \frac{1}{2} \mathbb{E} \sup _{t \in\left[0, k \wedge \tau_{N} \wedge \tilde{\zeta}_{K}\right]}\left(\|u\|_{H^{s}}^{2}+\|\rho\|_{H^{s}}^{2}\right)+e^{C_{K, k}} \mathbb{E} \int_{0}^{k}\left(\left\|\Phi_{1}\right\|_{\mathbb{H}^{s}}^{2}+\left\|\Phi_{2}\right\|_{\mathbb{H}^{s}}^{2}\right) d t .
\end{aligned}
$$

Taking the mathematical expectation in (4.6), we have

$$
\begin{aligned}
& \mathbb{E} \sup _{t \in\left[0, k \wedge \tau_{N} \wedge \widetilde{\zeta}_{K}\right]}\left(\|u\|_{H^{s}}^{2}+\|\rho\|_{H^{s}}^{2}\right)+\mathbb{E} \int_{0}^{k \wedge \tau_{N} \wedge \widetilde{\zeta}_{K}}\left(\|\nabla u\|_{H^{s}}^{2}+\|\nabla \rho\|_{H^{s}}^{2} d s\right) \\
& \leq e^{C_{K, k}} \mathbb{E}\left(\left\|u_{0}\right\|_{H^{s}}^{2}+\left\|\rho_{0}\right\|_{H^{s}}^{2}\right)+e^{C_{K, k}} \mathbb{E} \int_{0}^{k}\left(\left\|\Phi_{1}\right\|_{\mathbb{H}^{s}}^{2}+\left\|\Phi_{2}\right\|_{\mathbb{H}^{s}}^{2}\right) d t
\end{aligned}
$$

By the definition of $A_{N}^{k}$, one has

$$
A_{N}^{k} \subset\left\{\sup _{t \in\left[0, k \wedge \tau_{N} \wedge \tilde{\zeta}_{K}\right]}\left(\|u\|_{H^{s}}^{2}+\|\rho\|_{H^{s}}^{2}\right)+\int_{0}^{k \wedge \tau_{N} \wedge \tilde{\zeta}_{K}}\left(\|\nabla u\|_{H^{s}}^{2}+\|\nabla \rho\|_{H^{s}}^{2} d s\right) \geq N\right\}
$$

By Chebyshev's inequality, it follows from (4.9) that

$$
\mathbb{P}\left(A_{N}^{k}\right) \leq \frac{\exp \left(C_{K, k}\right)}{N} \mathbb{E}\left(\left\|u_{0}\right\|_{H^{s}}^{2}+\left\|\rho_{0}\right\|_{H^{s}}^{2}+\int_{0}^{k}\left(\left\|\Phi_{1}\right\|_{\mathbb{H}^{s}}^{2}+\left\|\Phi_{2}\right\|_{\mathbb{H}^{s}}^{2}\right) d t\right)
$$

Define $A^{k}=\left\{\tau \leq \zeta_{K}\right\} \cap\{\tau \leq k\}$, then $A^{k} \subset A_{N}^{k}$ for all $N$, so $\mathbb{P}\left(A^{k}\right)=0$. Since $\left\{\tau \leq \zeta_{K}\right\} \cap$ $\{\tau<\infty\}=\bigcup_{k=1}^{\infty} A^{k}$, then $\mathbb{P}\left(\left\{\tau \leq \zeta_{K}\right\} \cap\{\tau<\infty\}\right)=0$. The proof of Proposition 4.1 is thus complete.

Then, by the definition of $\zeta_{K}(1.3)$, we have $\widetilde{\zeta}_{K}=\zeta_{K}$ and $\zeta \leq \tau$ a.s. On the other hand, $\|u\|_{H^{s^{\prime}}} \leq\|u\|_{H^{s}}$, then $\left\|u\left(\tau_{N}\right)\right\|_{H^{s^{\prime}}} \leq N$. Due to the definition of $\zeta_{K}$, we have $\tau_{N} \leq \zeta_{N}$. Therefore,

$$
\tau=\zeta \quad \text { a.s., }
$$

and $\zeta$ is another blow-up time.

\subsection{The proof of (1.4)}

By Itô's formula for $\|\rho\|_{L^{2}}^{2}$, we have

$$
d\|\rho\|_{L^{2}}^{2}+2\|\nabla \rho\|_{L^{2}}^{2} d t=2 \sum_{i=1}^{\infty}\left\langle\rho, \Phi_{2, i}\right\rangle_{L^{2}} d \widetilde{W}_{i}+\left\|\Phi_{2}\right\|_{\mathbb{L}^{2}}^{2} d t
$$

By the Burkholder-Davis-Gundy inequality (2.4), we have

$$
\mathbb{E} \sup _{t^{\prime} \in\left[0, \zeta_{K} \wedge t\right]} 2 \sum_{i=1}^{\infty} \int_{0}^{t^{\prime}}\left\langle\rho, \Phi_{2, i}\right\rangle_{L^{2}} d \widetilde{W}_{i}
$$




$$
\begin{aligned}
& \leq C \mathbb{E}\left(\int_{0}^{t \wedge \zeta_{K}}\|\rho\|_{L^{2}}^{2}\left\|\Phi_{2}\right\|_{\mathbb{L}^{2}}^{2} d t\right)^{1 / 2} \\
& \leq \frac{1}{4} \mathbb{E} \sup _{t^{\prime} \in\left[0, \zeta_{K} \wedge t\right]}\|\rho\|_{L^{2}}^{2}+\mathbb{E} \int_{0}^{t}\left\|\Phi_{2}\right\|_{\mathbb{L}^{2}}^{2} d t .
\end{aligned}
$$

Therefore, we obtain

$$
\mathbb{E} \sup _{t^{\prime} \in\left[0, \zeta_{K} \wedge t\right]}\|\rho\|_{L^{2}}^{2} \leq C \mathbb{E}\left\|\rho_{0}\right\|_{L^{2}}^{2}+\mathbb{E} \int_{0}^{t}\left\|\Phi_{2}\right\|_{\mathbb{L}^{2}}^{2} d t .
$$

Applying Itô's formula for $\|u\|_{H^{s^{\prime}}}^{2}$, we have

$$
\begin{aligned}
d\|u\|_{H^{s^{\prime}}}^{2}+2\|\nabla u\|_{H^{s^{\prime}}}^{2} d t= & 2\langle u \cdot \nabla u, u\rangle_{H^{s^{\prime}}} d t+2\left\langle\rho e_{3}, u\right\rangle_{H^{s^{\prime}}} d t \\
& +2 \sum_{i=1}^{\infty}\left\langle\Phi_{1, i}, u\right\rangle_{H^{s^{\prime}}} d W_{i}+\left\|\mathbf{P} \Phi_{1}\right\|_{\mathbb{H}^{s^{\prime}}}^{2} d t .
\end{aligned}
$$

By (2.1), we bound

$$
\begin{aligned}
2\left|\langle u \cdot \nabla u, u\rangle_{H^{s^{\prime}}}\right| & \leq C\|u\|_{H^{s^{\prime}}}\|\nabla u\|_{H^{\frac{1}{2}}}\|u\|_{H^{s^{\prime}+1}} \\
& \leq C\|u\|_{H^{s^{\prime}}}^{s^{\prime}+\frac{1}{2}}\|u\|_{H^{s^{\prime}+1}}^{\frac{5}{2}-s^{\prime}} \\
& \leq \frac{1}{4}\|u\|_{H^{s^{\prime}+1}}^{2}+C\left(\|u\|_{H^{s^{\prime}}}^{\frac{4 s^{\prime}+2}{2 s^{\prime}}}+\|u\|_{H^{s^{\prime}}}^{2}\right),
\end{aligned}
$$

and

$$
\begin{aligned}
2\left|\left\langle\rho e_{3}, u\right\rangle_{H^{s^{\prime}}}\right| & \leq\|\rho\|_{L^{2}}\|u\|_{H^{2 s^{\prime}}} \\
& \leq \frac{1}{4}\|\nabla u\|_{H^{s^{\prime}}}^{2}+C\left(\|\rho\|_{L^{2}}^{2}+\|u\|_{H^{s^{\prime}}}^{2}\right) .
\end{aligned}
$$

Similar to (4.8), we have

$$
\begin{aligned}
& \sup _{t^{\prime} \in\left[0, t \wedge \zeta_{K}\right]} \sum_{i=1}^{\infty}\left|\int_{0}^{t^{\prime}}\left\langle\Phi_{1, i}, u\right\rangle_{H^{s^{\prime}}} d W_{i}\right| \\
& \leq \frac{1}{2} \mathbb{E} \sup _{t^{\prime} \in\left[0, t \wedge \zeta_{K}\right]}\|u\|_{H^{s^{\prime}}}^{2}+C \mathbb{E} \int_{0}^{t}\left\|\Phi_{1}\right\|_{\mathbb{H} s^{\prime}}^{2} d t .
\end{aligned}
$$

Combining (4.13)-(4.17) and recalling the definition of $\zeta_{K}$, we obtain

$$
\begin{aligned}
\mathbb{E} \sup _{t \in[0, \delta \wedge \zeta K]}\|u\|_{H^{s^{\prime}}}^{2}+\mathbb{E} \int_{0}^{\delta \wedge \zeta_{K}}\|\nabla u\|_{H^{s^{\prime}}}^{2} d t \\
\leq C\left(\mathbb{E}\left(\left\|u_{0}\right\|_{H^{s^{\prime}}}^{2}+\int_{0}^{\delta}\left\|\Phi_{1}\right\|_{\mathbb{H}^{s^{\prime}}}^{2} d t\right)+K^{1+\frac{2}{2 s^{\prime}-1} \delta+K \delta}\right. \\
\left.\quad+\delta \mathbb{E}\left(\left\|\rho_{0}\right\|_{L^{2}}^{2}+\int_{0}^{\delta}\left\|\Phi_{2}\right\|_{\mathbb{L}^{2}}^{2}\right)\right) \\
\leq C\left(K^{\left.1+\frac{1}{2 s^{\prime}-1} \delta+K \delta+\mathcal{A}(\delta)\right),}\right.
\end{aligned}
$$


where we define

$$
\mathcal{A}(\delta)=\mathbb{E}\left(\left\|u_{0}\right\|_{H^{s^{\prime}}}^{2}+\int_{0}^{\delta}\left\|\Phi_{1}\right\|_{\mathbb{H}^{s^{\prime}}}^{2} d t\right)+\delta \mathbb{E}\left(\left\|\rho_{0}\right\|_{L^{2}}^{2}+\int_{0}^{\delta}\left\|\Phi_{2}\right\|_{\mathbb{L}^{2}}^{2}\right)
$$

By the definition of $\zeta_{K}$, one has

$$
\left\{\omega \mid \zeta_{K} \leq \delta\right\} \subset\left\{\omega \mid \sup _{t \in\left[0, \delta \wedge \zeta_{K}\right]}\|u\|_{H^{s^{\prime}}}^{2} \geq K\right\}
$$

By Chebyshev's inequality, we obtain

$$
\mathbb{P}\left(\left\{\zeta_{K} \leq \delta\right\}\right) \leq \frac{C\left(K^{1+\frac{2}{2 s^{\prime}-1}} \delta+K \delta+\mathcal{A}(\delta)\right)}{K} .
$$

Let $\delta$ be given such that $0<\delta<1$. Choose an integer $K>0$ such that

$$
\frac{1}{K+1} \leq \delta^{\frac{2 s^{\prime}-1}{2 s^{\prime}+1}}<\frac{1}{K}
$$

then

$$
\mathbb{P}(\{\zeta>\delta\}) \geq \mathbb{P}\left(\left\{\zeta_{K}>\delta\right\}\right) \geq 1-C^{*} \delta^{\frac{2 s^{\prime}-1}{2 s^{\prime}+1}}(\mathcal{A}(\delta)+1) .
$$

\section{Acknowledgements}

I would like to thank my tutor Professor Ting Zhang for his suggestions which helped to greatly improve the paper.

\section{Funding}

This work is partially supported by Zhejiang Provincial Natural Science Foundation of China LR17A010001, National Natural Science Foundation of China 11771389.

Availability of data and materials

Not applicable.

\section{Competing interests}

The author declares that they have no competing interests.

\section{Author's contributions}

The author read and approved the final manuscript.

\section{Publisher's Note}

Springer Nature remains neutral with regard to jurisdictional claims in published maps and institutional affiliations.

Received: 1 May 2018 Accepted: 18 February 2019 Published online: 26 February 2019

\section{References}

1. Constantin, P., Doering, C.R.: Infinite Prandtl number convection. J. Stat. Phys. 94(1-2), 159-172 (1999)

2. Gill, A.: Atmosphere-Ocean Dynamics. International Geophysics Series. Academic Press, London (1982)

3. Majda, A.: Introduction to PDEs and Waves for the Atmosphere and Ocean. Courant Lecture Notes in Mathematics, vol. 9 (2003)

4. Pedlosky, J.: Geophysical Fluid Dynamics. Springer, Berlin (2013)

5. Adhikari, D., Cao, C., Wu, J.: Global regularity results for the 2D Boussinesq equations with vertical dissipation. J. Differ. Equ. 251(6), 1637-1655 (2011)

6. Chae, D.: Global regularity for the 2D Boussinesq equations with partial viscosity terms. Adv. Math. 203(2), 497-513 (1997)

7. Hou, T.Y., Li, C.: Global well-posedness of the viscous Boussinesq equations. Discrete Contin. Dyn. Syst. 12(1), 1-12 (2005)

8. Lai, M.-J., Pan, R., Zhao, K.: Initial boundary value problem for two-dimensional viscous Boussinesq equations. Arch. Ration. Mech. Anal. 199(3), 739-760 (2011)

9. $\mathrm{Wu}, J ., \mathrm{Xu}, \mathrm{X}$., Ye, Z.: The $2 \mathrm{D}$ Boussinesq equations with fractional horizontal dissipation and thermal diffusion. J. Math. Pures Appl. 115(9), 187-217 (2018) 
10. Adhikari, D., Cao, C., Shang, H., Wu, J., Xu, X., Ye, Z.: Global regularity results for the 2D Boussinesq equations with partial dissipation. J. Differ. Equ. 260(2), 1893-1917 (2016)

11. Yang, W., Jiu, Q., Wu, J.: Global well-posedness for a class of 2D Boussinesq systems with fractional dissipation. J. Differ Equ. 257(11), 4188-4213 (2014)

12. $\mathrm{Wu}, \mathrm{J}$., $\mathrm{Xu}, \mathrm{X} ., \mathrm{Xue}, \mathrm{L} ., \mathrm{Ye}, \mathrm{Z}$.: Regularity results for the $2 \mathrm{D}$ Boussinesq equations with critical or supercritical dissipation. Commun. Math. Sci. 14(7), 1963-1997 (2016)

13. Jiu, Q., Miao, C., Wu, J., Zhang, Z.: The $2 \mathrm{D}$ incompressible Boussinesq equations with general critical dissipation. SIAM J. Math. Anal. 46, 3426-3454 (2014)

14. Ye, Z:: Global smooth solution to the $2 \mathrm{D}$ Boussinesq equations with fractional dissipation. Math. Methods Appl. Sci. 40(12), 4595-4612 (2017)

15. Ye, Z., Xu, X.: Global well-posedness of the 2D Boussinesq equations with fractional Laplacian dissipation. J. Differ. Equ. 260(8), 6716-6744 (2016)

16. Chae, D., Nam, H.-S.: Local existence and blow-up criterion for the Boussinesq equations. Proc. R. Soc. Edinb., Sect. A 127(5), 935-946 (1997)

17. Danchin, R., Paicu, M.: Existence and uniqueness results for the Boussinesq system with data in Lorentz spaces. Physica D 237(10-12), 1444-1460 (2008)

18. Fan, J., Sun, W., Yin, J.: Blow-up criteria for Boussinesq system and MHD system and Landau-Lifshitz equations in a bounded domain. Bound. Value Probl. 2016, 90 (2016)

19. Fan, J., Zhou, Y.: A note on regularity criterion for the 3D Boussinesq system with partial viscosity. Appl. Math. Lett. 22(5), 802-805 (2009)

20. Jiu, Q., Yu, H.: Global well-posedness for 3D generalized Navier-Stokes-Boussinesq equations. Acta Math. Appl. Sin. Engl. Ser. 32(1), 1-16 (2016)

21. Ye, Z: A logarithmically improved regularity criterion of smooth solutions for the 3D Boussinesq equations. Osaka J. Math. 53(2), 417-423 (2016)

22. Ferrario, B.: The Bénard problem with random perturbations: dissipativity and invariant measures. NoDEA Nonlinear Differ. Equ. Appl. 4(1), 101-121 (1997)

23. Pu, X., Guo, B.: Global well-posedness of the stochastic 2D Boussinesq equations with partial viscosity. Acta Math. Sci. Ser. B Engl. Ed. 31(5), 1968-1984 (2011)

24. Duan, J., Millet, A.: Large deviations for the Boussinesq equations under random influences. Stoch. Process. Appl. 119(6), 2052-2081 (2009)

25. Brzeźniak, Z., Motyl, E.: The existence of martingale solutions to the stochastic Boussinesq equations. Glob. Stoch. Anal. 1(2), 175-216 (2011)

26. Yamazaki, K:: Global martingale solution for the stochastic Boussinesq system with zero dissipation. Stoch. Anal. Appl. 34(3), 404-426 (2016)

27. Kim, J.U.: Strong solutions of the stochastic Navier-Stokes equations in $\mathbb{R}^{3}$. Indiana Univ. Math. J. 59(4), 1417-1450 (2010)

28. Da Prato, G., Zabczyk, J.: Stochastic Equations in Infinite Dimensions, 2nd edn. Encyclopedia of Mathematics and Its Applications, vol. 152. Cambridge University Press, Cambridge (2014)

29. Evans, L.C.: An Introduction to Stochastic Differential Equations. Am. Math. Soc., Providence (2013)

30. Karatzas, I., Shreve, S.E.: Brownian Motion and Stochastic Calculus, 2nd edn. Graduate Texts in Mathematics, vol. 113 Springer, New York (1991)

31. Kuo, H.-H.: Introduction to Stochastic Integration. Universitext. Springer, New York (2006)

\section{Submit your manuscript to a SpringerOpen ${ }^{\circ}$ journal and benefit from:}

- Convenient online submission

- Rigorous peer review

- Open access: articles freely available online

- High visibility within the field

- Retaining the copyright to your article

Submit your next manuscript at $\gg$ springeropen.com 